\title{
Comportamento Altruísta na Infância: O que a Literatura nos Mostra*
}

\author{
Suellen Vilalva ${ }^{1,2, * *}$ (D, Suzane Schmidlin $\operatorname{Löhr}^{2}$ @, \& Matheus Guedes ${ }^{2,3}$ (D) \\ ${ }^{1}$ Faculdade Herrero, Curitiba, PR, Brasil \\ ${ }^{2}$ Universidade Federal do Paraná, Curitiba, PR, Brasil \\ ${ }^{3}$ Universidade Estadual do Centro-Oeste, Guarapuava, PR, Brasil
}

\begin{abstract}
RESUMO - Uma variedade de comportamentos altruístas diferenciados tem sido evidenciada na infância. O presente artigo objetivou sistematizar estudos empíricos produzidos nos anos de 2010 a 2015 sobre o altruísmo infantil. Após a elaboração e aplicação de um protocolo sistematizado de seleção, foram encontrados 14 artigos, os quais foram analisados na íntegra. Os estudos contemplaram o altruísmo infantil ( 1 a 11 anos de idade) em associação a diferentes variáveis, tais como avaliação moral, custos de resposta, renda familiar, distância social, capacidade de abster-se de prêmios imediatos, eventos adversos, representações de apego, dominância social, composição genética, entre outras. Concluiu-se que crianças ainda muito jovens em contextos variados demonstram altruísmo por meio de ações topograficamente diversas à medida que encontram oportunidades para isso.
\end{abstract}

PALAVRAS-CHAVE: revisão sistemática, altruísmo infantil, comportamento altruísta

\section{Altruistic Behavior in Childhood: What does Literature Shows us}

\begin{abstract}
A variety of altruistic behaviors differentiated has been evidenced in childhood. This article aimed to systematize empirical studies produced in the years 2010 to 2015 about child altruism. After the elaboration and application of a systematic selection protocol, were found 14 articles were fully analyzed. The studies have examined the child altruism ( 1 to 11 years old) in association with different variables such as moral evaluation, response costs, family income, social distance, ability to abnegate immediate awards, adverse events, representations of attachment, social dominance, genetic composition, among others. It was concluded that children still very young and in different contexts demonstrate altruism through different topographic actions if they find opportunities for this.
\end{abstract}

KEYWORDS: systematic review, child altruism, altruistic behavior

Viver é comportar-se. O ser humano, enquanto vive, comporta-se tanto individualmente quanto em grupo. As interações ocasionadas pela ação de um ou mais indivíduos em relação a outros são definidas como comportamentos sociais. E, como tais, ocorrem em um ambiente social que não pode ser desprezado em virtude das variáveis que o compõem e que influenciam diretamente o padrão de comportamento dos seres humanos (Skinner, 1979/2000). A sociedade é produto das relações sociais (Skinner, 1979/2000) e "o comportamento social surge porque um organismo é importante para outro como parte de seu ambiente" (Skinner, 1979/2000, p. 326). Os benefícios de viver em grupo só se tornaram possíveis devido à existência de mecanismos que tornam os seres humanos sensíveis uns aos outros (Baum, 2006).

Em situações nas quais os indivíduos precisam decidir de que forma irão comportar-se, eles recorrem a um conjunto de normas culturalmente estabelecidas e selecionam aquelas que julgam mais apropriadas de serem cumpridas (Vásquez, 1995), pautando sobre elas suas ações. A seleção

* O referido artigo é baseado na Dissertação de Mestrado da primeira autora.

** E-mail: suellen.vila@gmail.com

— Submetido: 20/02/2020; Revisado: 04/03/2020; Aceito: 27/05/2020. 
de quais regras seguir associa-se diretamente à história de aprendizagem individual e coletiva do ser humano, uma vez que os diferentes contextos de seu desenvolvimento, além de conter regras de convivência, também estabelecem reforço ou medidas de extinção de condutas específicas (Ferreira $\&$ Carrara, 2009).

As decisões individuais ou coletivas sempre estiveram atreladas a diretrizes e normativas que privilegiaram a convivência e a sobrevivência humana (Santos, 2017; Silva \& Moreira, 2019). Tal reflexão pertence ao campo da ética e da moral, no qual as regras inscritas como leis, ao descreverem um conjunto de normas, frequentemente levam a sociedade a rotular alguns comportamentos como "bons" e "maus", de forma que uma dada cultura irá então definir quais comportamentos são aceitáveis ou condenáveis, instituindo assim um conjunto de valores sob os quais se dará o funcionamento característico daquele grupo (Baum, 2006; Santos, 2017).

No entanto, para muito além do juízo moral entre bom ou mal, é necessário compreender a questão valorativa presente no dia a dia da cultura relacionada à função dos comportamentos sociais moralmente relevantes para a vida em sociedade. Valorar condutas como cooperação, o cuidado e a solidariedade têm uma função adaptativa para a espécie, uma vez que elas contribuem para a manutenção das relações sociais (Rex, 2016). Alguns autores destacam que, frente a problemas sociais, tais como miséria, pobreza e práticas violentas, os comportamentos de natureza altruísta seriam uma alternativa resolutiva (Guimarães \& Laurenti, 2019).

$\mathrm{O}$ altruísmo vem sendo conceituado de inúmeras e distintas maneiras (Rodrigues et al., 1999; Batson \& Powell, 2003; Chou, 1996; Krueger et al., 2001; Lencastre, 2010; Moñivas, 1996; Schlinger, 1995; Sober \& Wilson, 1999). As definições se alteram de acordo com diferentes variáveis, uma heterogeneidade que dificulta a definição precisa do termo e, consequentemente, a classificação operacional de condutas dessa natureza. Uma das tentativas menos subjetiva de conceituação da terminologia surgiu do estudo de Vilalva e Lohr (2019, p. 151), que buscaram compreender o altruísmo enquanto comportamento aprendido:

\footnotetext{
"Tendo em vista a complexidade de definições existentes, nesse estudo, com base em Schlinger (1995), o altruísmo será compreendido como uma subclasse de comportamento moral, a qual incluirá ações nas quais um indivíduo se mobiliza para prestar algum tipo de auxílio a outro ou a outros, com a finalidade de fazer cessar ou reduzir sua tensão, angústia ou mal-estar - esta é sua função. Tais ações poderão ser manifestas de diferentes formas - esta é, então, sua topografia. Podem ser considerados como altruístas comportamentos como cooperação, partilha, consolo, entre outros."
}

O altruísmo tem sido admitido como a marca registrada de uma sociedade, o qual, em longo prazo, além de propiciar benefícios grupais, confere vantagens aos indivíduos que fazem parte desse grupo (Baum, 2006). Os benefícios de comportamentos de natureza moral, especificamente aqueles considerados altruístas ou pró-sociais, são encontrados em uma série de estudos, sobretudo no que diz respeito à manutenção das relações (Allen-Arave et al., 2008; De Backer et al., 2015; Ostrov \& Guzzo, 2015; Schmidt \& Sommerville, 2011; Warneken \& Tomasello, 2011), em razão de o altruísmo ser considerado antagônico ao egoísmo. Há indicações de que, do ponto de vista biológico, indivíduos com tendências altruístas teriam as atividades da amígdala aumentadas em comparação a indivíduos sociopatas, admitindo-se que, em uma escala de cuidado com o ser humano, em um extremo, estariam localizados indivíduos com tendências altruístas e altruístas extremas e, no outro, indivíduos com tendências a comportamentos antissociais (Nahra, 2016).

Ao considerar o altruísmo como um comportamento passível de aprendizagem, é necessário considerar a infância como um período de significativas aprendizagens, pois é nessa etapa que a criança, por meio de suas interações, irá estabelecer seus primeiros referenciais de como comportarse em diferentes contextos. Assim, as crianças tenderão a engajar-se de forma crescente e contínua em interações sociais complexas (Bijou, 1995; Schlinger, 1995) que cada vez mais lhe exigirão novas aquisições comportamentais morais. Pesquisas sugerem a existência de uma habilidade infantil de discriminar diferentes pistas sociais na tomada de decisão de comportar-se altruisticamente, sobretudo, se há possibilidade de atribuição de mérito pela exibição do cuidado para com o outro (Leimgruber et al., 2012; Sampaio \& Carmino, 2017).

Há evidências de que, antes dos 2 anos de idade, crianças podem apresentar um senso de justiça que influenciará diretamente suas ações e, a depender dos estímulos contextualmente recebidos, elas podem vir a comportar-se de forma altruísta (Barragan \& Dweck, 2014). Além disso, a aprendizagem de comportamentos altruístas também é apontada como preventiva a desajustamentos comportamentais, uma vez que comportamentos dessa natureza podem diminuir comportamentos incompatíveis, como, por exemplo, comportamentos antissociais (Sabbag, 2017). Pesquisas também destacam que a empatia é o elo para o comportamento altruísta, assim, crianças empáticas e capazes de mobilizar-se em prol de outrem podem apresentar maior desenvoltura social e menores índices de problemas de comportamento ao longo da infância (Flouri \& Sarmadi, 2015).

Embora os comportamentos sociais de natureza moral possam assumir diferentes topografias, isto é, possam se apresentar de diferentes formas assumindo funcionalidades distintas (Dias, 2016), este artigo focará especialmente o comportamento altruísta, de forma que o objetivo desse estudo é, por meio de revisão sistemática da literatura, investigar a conduta altruísta em crianças, levando em consideração diversas variáveis contextuais relacionadas à aquisição e manutenção do comportamento altruísta. 


\section{MÉTODO}

Trata-se de um estudo de revisão sistemática da literatura, que assume caráter exploratório descritivo acerca do altruísmo em relação à conduta infantil. $O$ estudo teve a seguinte questão norteadora: $\mathrm{O}$ altruísmo em relação à conduta infantil tem sido contemplado como tema de estudos empíricos?

\section{Procedimentos de Busca e de Seleção}

Inicialmente, os descritores (palavras-chave) altruísmo e comportamento altruista foram submetidos a consulta no Thesaurus ${ }^{1}$ da Biblioteca Virtual em Saúde (BVS) (http:// goo.gl/ZHzI56) e Thesaurus Brasileiro da Educação (INEP) (http://goo.gl/rb99qL). O Thesaurus da BVS associou o descritor altruísmo a pesquisas na área, entretanto, não associou o descritor comportamento altruísta a nenhuma pesquisa anterior. Já o Thesaurus do INEP não forneceu nenhuma associação de ambos os descritores a pesquisas preexistentes.

A etapa seguinte, nomeada como $1^{\text {a }}$ fase de busca, consistiu no levantamento bibliográfico geral nas bases de dados Biblioteca Virtual em Saúde (BVS) e Periódicos CAPES (CAPES), nas quais foram inseridos os descritores já mencionados. Tais bases de dados foram escolhidas pela multidisciplinaridade de suas publicações, logo, pela riqueza de conteúdos que se supõe estar a elas indexados. O resultado geral, ou seja, sem o estabelecimento de nenhum critério, na data de 7 de março de 2016, foi de 6.051 documentos, conforme ilustrado na Tabela 1.

Na segunda fase de busca, para ambas as bases (BVS e CAPES), foram estabelecidas as seguintes estratégias: (1) Inserção dos descritores em letras maiúsculas e em língua portuguesa; (2) Seleção de materiais publicados nos últimos 05 anos; (4) Filtragem por "tipo de recurso/tipo de documento": artigos. Nessa etapa, foram encontrados 1.504 artigos, sendo 1.418 artigos da BVS (altruísmo $=$ 1.417 / comportamento altruista $=1)$ e 86 artigos da CAPES (altruísmo $=81 /$ comportamento altruísta $=5$ ).

$\mathrm{Na}$ terceira fase de busca, os critérios estabelecidos se diferenciaram devido à peculiaridade dos filtros contidos em cada uma das bases. Assim, em cada uma delas, foram utilizados filtros específicos. Na base de dados BVS, utilizamos: (1) Texto completo: disponível; (2) Assunto principal: Altruísmo; (3) Limite: criança/ criança préescolar/ lactente/ recém-nascido; (4) Tipo de documento: artigo; (5) Idioma: português e inglês. $\mathrm{Na}$ base de dados CAPES, utilizamos: (1) Tipo de documento: artigo; (2) Seleção de materiais publicados no período de 2011 a 2016;

$1 \mathrm{O}$ Thesaurus pode ser compreendido como um glossário que reúne termos escolhidos a partir de uma matriz conceitual previamente elaborada e destinada à indexação de documentos e informações num determinado campo do saber (Inep, 2011).
(3) Idioma: português e inglês. Todos os filtros mencionados foram selecionados simultaneamente em cada uma das bases de dados. Resultaram, então, 74 artigos, 40 da base BVS (altruismo $=40$ /comportamento altruista $=0$ ) e 34 da base CAPES (altruísmo $=33 /$ comportamento altruísta $=1$ ).

Após terem sido identificados 74 artigos para análise, esses foram submetidos ao teste de relevância, adaptado de Azevedo (2010), incluindo apenas aqueles que obtivessem respostas afirmativas aos seguintes questionamentos: 1) O artigo em questão traz resumo completo? 2) Descreve o altruísmo e/ou comportamentos de natureza moral similar em relação à conduta da criança? 3) É um estudo de natureza empírica?

Foram obtidos 14 artigos, sendo 13 oriundos da base de dados BVS e 1 da base de dados CAPES. A Figura 1 apresenta resumidamente as fases de busca e os respectivos números encontrados.

O processo de busca, seleção e avaliação dos artigos foi realizado por dois juízes simultaneamente, a fim de extinguir qualquer tendenciosidade, conforme recomenda a literatura (Koller et al., 2004). Tais juízes eram psicólogos e pesquisadores com conhecimento prévio na temática do altruísmo e possuíam fluência na leitura da língua portuguesa e inglesa. Dos 74 artigos selecionados submetidos ao teste de relevância, 59 foram excluídos por não atenderem aos critérios preestabelecidos. Ocorre ainda que alguns artigos se repetiram mais de uma vez em uma única base, sendo computados apenas uma vez ao longo do estudo.

\section{Resultados e Discussão}

Os 14 artigos analisados, os quais resultaram de todo o processo de filtragem, foram publicados nos periódicos: PloS One $(\mathrm{n}=4)$, APS (Association for Psychological Science) $(n=2)$, Journal of Experimental Child Psychology $(n=2)$, Cognitive Processing $(n=1)$, Cognitive Science $(n=1)$, The Journal of Genetic Psychology ( $\mathrm{n}=1$ ), PNAS (Proceedings of the National Academy of Sciences of the United States of America) ( $\mathrm{n}=1)$, Psico-USF ( $\mathrm{n}=1)$, Development and Psychopathology $(\mathrm{n}=1)$. As buscas indicaram maior quantidade de artigos publicados nos anos de $2013(\mathrm{n}=5)$, $2015(n=3)$ e $2011(n=3)$. Nos demais anos, o número de publicações ficou entre um e dois artigos. Dentre as áreas do conhecimento dos periódicos em que os artigos analisados foram publicados, destacaram-se os eixos multidisciplinar e psicológico.

A grande maioria dos estudos $(n=13)$ caracterizaramse como estudos de corte transversal, havendo apenas 1 estudo longitudinal (Li, Li, Decety \& Lee, 2013). Os artigos eram predominantemente de língua inglesa, sendo apenas um de língua portuguesa (Bartholomeu, Silva \& Montiel, 2011). Os procedimentos e/ou recursos utilizados 

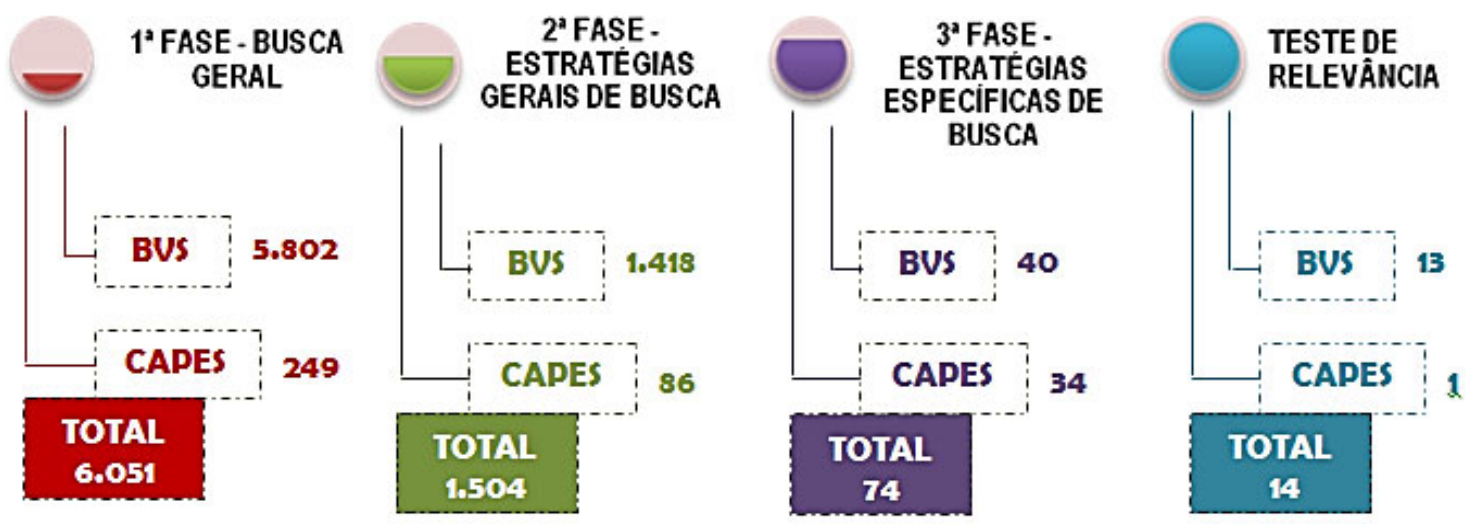

Figura 1. Fases do procedimento de busca e seleção.

Tabela 1

Distribuição geral de documentos de acordo com as bases bibliográficas.

\begin{tabular}{cccc}
\hline DESCRITORES ASSOCIADOS & BVS & CAPES & *TOTAL GERAL \\
\hline ALTRUÍSMO & 5.795 & 233 & 6.028 \\
COMPORTAMENTO ALTRUÍSTA & 7 & 16 & 23 \\
TOTAL & 5.802 & 249 & $\mathbf{6 . 0 5 1}$ \\
\hline
\end{tabular}

nos estudos envolveram tarefas nas quais as crianças tiveram a oportunidade de fornecer algum tipo de ajuda $(n=1)$; tarefas de alocação de recursos $(n=9)$; entrevistas utilizando historietas $(\mathrm{n}=2)$; observações de comportamento em ambiente escolar e entrevista do docente responsável pela criança $(n=1)$; escala padronizada para verificação de habilidades sociais $(\mathrm{n}=1)$. Acerca da faixa etária, a idade dos participantes dos estudos variou entre 1 e 11 anos. Os temas de investigação contemplados nos estudos foram: doação, partilha, dominância social, confiabilidade, cooperação e ajuda. A Tabela 2 fornece uma síntese dos artigos selecionados e analisados:

Em relação à temática, dentre os artigos resultantes do levantamento sistemático $(\mathrm{n}=14)$, foram incluídos dois artigos que não abordavam propriamente o comportamento altruísta, contudo, atendiam aos critérios preestabelecidos no teste de relevância. Um deles foi o estudo de $\mathrm{Fu}$ et al. (2014), que narrou a capacidade das crianças de confiar não somente em indivíduos que dizem a verdade, mas também naqueles que mentem para beneficiar o próximo. Esse foi incluído por investigar a confiança como um comportamento de natureza moral. O outro estudo foi o de Bartholomeu et al. (2011), que descreveu o desenvolvimento de uma medida de avaliação das habilidades infantis e seus índices de precisão para o uso em pesquisas posteriores. Esse foi incluído em razão de uma das dimensões do instrumento ser nomeada como Civilidade e Altruísmo.

Os demais estudos abordam o altruísmo enfatizando diferentes classes comportamentais, tais como: ajuda, partilha, doação e cooperação (Aguilar-Pardo et al., 2013; Avinun et al., 2011; Bakermans-Kranenburg \& Ijzendoorn, 2011; Barragan \& Dweck, 2014; Chen et al., 2013; Chernyak \& Kushnir, 2013; Leimgruber et al., 2012; Li et al., 2013; Ostrov \& Guzzo, 2015; Schmidt \& Sommerville, 2011; Sebastián-Enesco \& Warneken, 2015). Como critério para classificar e agrupar os comportamentos altruístas investigados, foi utilizada a própria nomenclatura empregada pelos autores em cada um dos artigos analisados.

O comportamento de ajuda refere-se a contribuir com outro indivíduo para que este faça alguma coisa (Ferreira, 1999), nesse o objetivo final da ação não é necessariamente comum a ambas as partes, ajudado e ajudador. Já o comportamento de cooperação diz respeito a agir ou trabalhar junto com outro ou outros para um fim comum (Weiszflog, 2004), ou seja, o objetivo final da ação contempla ambas as partes. Nos estudos referentes aos comportamentos de partilha, fica expressa a possibilidade de a criança alocar recursos dos quais dispõe para um outro indivíduo. Contudo, esta mesma operação é nomeada por alguns autores como doação (Avinun et al., 2011; Bakermans-Kranenburg \& Ijzendoorn, 2011; Chen et al., 2013; Fu et al., 2014; Leimgruber et al., 2012; Li et al., 2013), enquanto por outros como partilha (Schmidt \& Sommerville, 2011; Sebastián-Enesco \& Warneken, 2015), e há casos ainda em que um mesmo artigo faz uso de ambos os termos para se referir à mesma ação (Aguilar-Pardo et al., 2013; Chernyak \& Kushnir, 2013). 


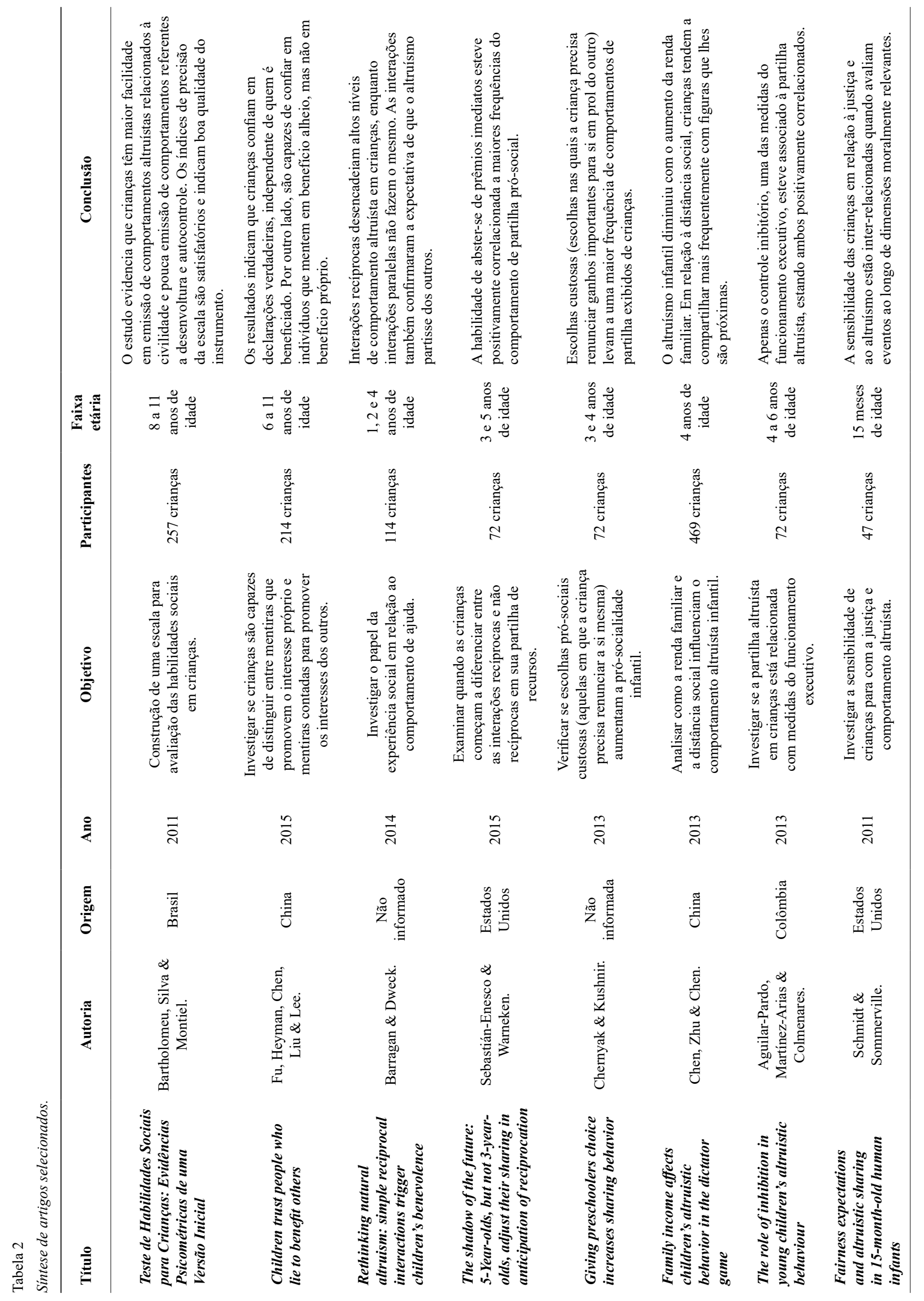




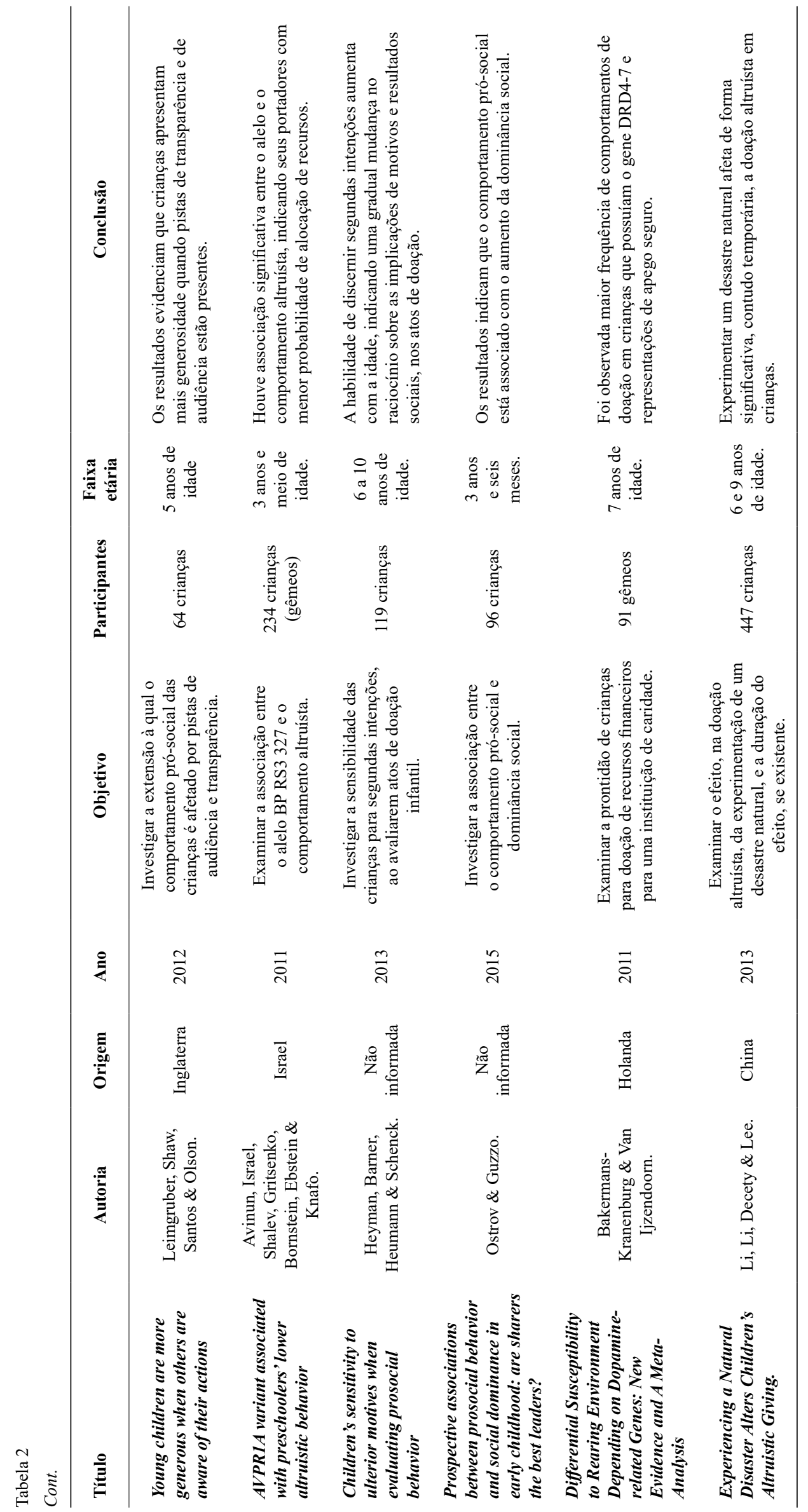


Ao investigarem a associação entre comportamento pró-social (partilha, ajuda, colaboração, entre outros) e dominância social, Ostrov e Guzzo (2015) evidenciaram que o comportamento pró-social infantil esteve associado com o aumento da dominância social. Ou seja, as crianças classificadas como melhores líderes eram aquelas que partilhavam, ajudavam e incluíam os outros de forma crescente em suas escolhas pró-sociais. No que se refere ao comportamento de ajuda, Barragan e Dweck (2014), ao investigarem o papel da experiência social em relação ao altruísmo, concluíram que as interações recíprocas às quais as crianças foram expostas desencadearam altos níveis de comportamento altruísta, enquanto interações paralelas não resultaram no mesmo efeito.

Dentre os estudos focados no comportamento de partilha, Sebastián-Enesco e Warneken (2015) identificaram que crianças com 3 anos de idade apresentaram dificuldades de prever oportunidades de retribuição futura. Já crianças de 5 anos foram capazes de raciocinar levando em consideração as oportunidades de retribuição futura, e assim, estrategicamente modularam seus comportamentos de partilha de forma recíproca. Além disso, a habilidade infantil de adiar a gratificação foi associada a maior frequência de partilha pró-social, uma vez que exigiu que crianças renegassem os próprios interesses em prol de uma gratificação futura. Corroborando tais achados, Aguilar-Pardo, Martínez-Arias e Colmenares (2013) ${ }^{2}$, ao investigarem a associação entre partilha e controle inibitório, afirmaram que a capacidade das crianças de abster-se de ganhos pessoais ou de propor injustas alocações de recursos está diretamente associada com a variação do comportamento altruísta. Assim, no que diz respeito ao funcionamento executivo, apenas o controle inibitório esteve associado à partilha altruísta, estando ambos positivamente correlacionados. Nessa mesma direção, o estudo de Chernyak e Kushnir (2013) ${ }^{3}$ indicou que dar às crianças oportunidades de escolhas pró-sociais custosas (escolhas nas quais a criança precisa abrir mão de ganhos importantes para si em prol do outro) desempenha um importante papel em seu comportamento pró-social subsequente, uma vez que escolhas custosas ocasionaram uma maior frequência de comportamentos de partilha exibidos posteriormente pelas crianças. Schmidt e Sommerville (2011) verificaram sinais de um senso de justiça por parte das crianças que demonstravam expectativas de que a alocação de recursos fosse equilibrada entre parceiros. Os autores relacionaram tal senso de justiça com o altruísmo.

Alguns estudos centraram-se no comportamento de doação (Avinun et al., 2011; Chen et al., 2013; Leimgruber et al., 2012; Ostrov \& Guzzo, 2015). Os pesquisadores Chen, Zhu e Chen (2013) concluíram que o altruísmo infantil diminuiu com o aumento da renda familiar e que crianças

2 Tais autores utilizam sinonimamente os termos partilha e doação. 3 Tais autores utilizam sinonimamente os termos partilha e doação. doaram mais recursos a amigos do que a estranhos, sugerindo que comportamentos pró-sociais direcionados a amigos e estranhos podem diferir desde a infância. Leimgruber et al. (2012) demonstraram que crianças podem comportar-se de acordo com padrões adultos de pró-socialidade, sendo mais generosas em suas doações quando expostas a pistas de transparência e de audiência. Heyman et al. (2013) apontaram para a capacidade moral avaliativa das crianças, indicando que elas podem ter dificuldades de compreender segundas intenções devido a um lento processo de mudança de raciocínio infantil acerca de implicações motivacionais relacionadas a resultados sociais.

Li et al. (2013) examinaram o efeito da experimentação de um desastre natural na doação altruísta infantil, concluindo que crianças com 9 anos de idade e altos níveis empáticos ques haviam vivenciado um terremoto eram capazes imediatamente de doar mais recursos se comparadas a crianças de 6 anos de idade. Contudo, tal efeito não se manteve em longo prazo.

A natureza biológica e cultural do comportamento altruísta foi analisada por Bakermans-Kranenburg e Ijzendoorn (2011), que descobriram uma interação significativa do alelo de repetição DRD4-7 (fator biológico) com o apego seguro (fator cultural). Tal associação esteve relacionada com a maior doação infantil de recursos. $\mathrm{Ou}$ seja, ambos os determinantes do comportamento altruísta de doação, tanto genético quanto ambiental, foram apontados como relevantes, contudo, somente quando em interação. Já Avinun et al. (2011) deram maior ênfase ao fator biológico e descreveram que a presença do alelo AVPR1A RS3 327 esteve associado com uma menor frequência de doação de recursos.

Em relação à faixa etária, Barragan e Dweck (2014) apontaram que, antes dos 2 anos de idade, as crianças apresentam um senso de justiça que influencia diretamente suas ações. Já aos 3 anos elas são capazes de comportarse de acordo com padrões adultos de pró-socialidade, expressando maior generosidade quando suas ações são visíveis a terceiros. Além disso, crianças que expressam padrões altruístas de comportamento tendem a ser vistas como melhores líderes (Leimgruber et al., 2012; Ostrov \& Guzzo, 2015). E, embora dos 3 aos 6 anos de idade possa haver uma tendência egoísta conflitante no que diz respeito à manifestação de comportamentos pró-sociais, sobretudo aqueles que são custosos, há evidências de que, ainda nessa idade, crianças podem demonstrar disposição em compartilhar seus recursos, ainda que isso exija que renunciem a seus desejos para beneficiar outrem (Aguilar-Pardo et al., 2013; Chernyak \& Kushnir, 2013; Sebastián-Enesco \& Warneken, 2015). Estudos com crianças dos 4 aos 11 anos mostraram que variáveis como renda familiar, distância social, capacidade de abster-se de prêmios imediatos, eventos adversos, representações de apego e composição genética foram associadas ao comportamento altruísta (Aguilar-Pardo et al., 2013; Avinun et al., 2011; 
Bakermans-Kranenburg \& Ijzendoorn, 2011; Chen et al., 2013; Chernyak \& Kushnir, 2013; Li et al., 2013; Schmidt \& Sommerville, 2011; Sebastián-Enesco \& Warneken, 2015).

No que diz respeito à cultura, os estudos elegidos foram realizados com participantes de culturas distintas (AguilarPardo et al., 2013; Bakermans-Kranenburg \& Ijzendoorn, 2011; Leimgruber et al., 2012; Schmidt \& Sommerville, 2011; Sebastián-Enesco \& Warneken, 2015). Em termos geográficos, seis deles incluíram crianças oriundas de culturas ocidentais, quatro de culturas orientais e outros quatro não indicaram a cultura em que foram desenvolvidos. Os estudos realizados em culturas ocidentais dedicaramse a investigar comportamentos de partilha e doação (Aguilar-Pardo et al., 2013; Bakermans-Kranenburg \& Ijzendoorn, 2011; Leimgruber et al., 2012; Schmidt \& Sommerville, 2011; Sebastián-Enesco \& Warneken, 2015) enquanto os estudos orientais se dedicaram a investigar os comportamentos infantis de confiança e doação. Percebe-se, como ponto em comum nos estudos realizados em ambas as culturas, a investigação de comportamentos de doação. Além disso, tanto nas culturas ocidentais como nas orientais houve uma preocupação com a investigação de fatores genéticos em relação ao altruísmo.

Em síntese, os artigos analisados indicaram que, desde a primeira infância, as crianças são capazes de exibir comportamentos altruístas, tais como partilha, doação, ajuda, colaboração, entre outros comportamentos pró-sociais (Aguilar-Pardo et al., 2013; Avinun et al., 2011; BakermansKranenburg \& Ijzendoorn, 2011; Barragan \& Dweck, 2014; Chen et al., 2013; Chernyak \& Kushnir, 2013; Leimgruber et al., 2012; Li et al., 2013; Ostrov \& Guzzo, 2015; Schmidt \& Sommerville, 2011; Sebastián-Enesco \& Warneken, 2015).

$\mathrm{O}$ raciocínio moral adjacente às avaliações morais $\mathrm{e}$ escolhas pró-sociais da criança foi apontado nas faixas etárias de um ano, cinco anos e dos seis aos onze anos de idade (Fu et al., 2014; Leimgruber et al., 2012; Schmidt \& Sommerville, 2011). Além disso, crianças apresentam sinais de senso de justiça desde o primeiro ano de idade e tal senso de justiça contribui positivamente para o desempenho altruísta infantil (Schmidt \& Sommerville, 2011). Diferentes variáveis foram contempladas em associação ao altruísmo nos estudos selecionados, tais como: visibilidade de terceiros em relação a suas ações, custo de resposta, renda familiar, distância social, capacidade de abster-se de prêmios imediatos, eventos adversos, representações de apego e composição genética, entre outras (Aguilar-Pardo et al., 2013; Avinun et al., 2011; Bakermans-Kranenburg \& Ijzendoorn, 2011; Chen et al., 2013; Chernyak \& Kushnir, 2013; Leimgruber et al., 2012; Li et al., 2013; Ostrov \& Guzzo, 2015; Schmidt \& Sommerville, 2011; SebastiánEnesco \& Warneken, 2015).

Evidenciaram-se como elementos fundamentais presentes durante a manifestação do comportamento altruísta as interações recíprocas (Barragan \& Dweck, 2014), por meio das quais as crianças têm a oportunidade de engajar-se em comportamentos pró-sociais de forma casual ou estratégica (Sebastián-Enesco \& Warneken, 2015). O comportamento altruísta infantil também é apontado como preditor de dominância social (Ostrov \& Guzzo, 2015). Contudo, comportar-se de maneira altruísta pode exigir que as crianças reneguem os próprios interesses, indicando-se que é, em face a esse exercício, que o altruísmo pode ser incorporado ao repertório de comportamentos infantis (Aguilar-Pardo et al., 2013; Sebastián-Enesco \& Warneken, 2015). Crianças podem ainda comportar-se de acordo com padrões adultos de pró-socialidade, sendo mais generosas quando suas ações são visíveis. Ademais, ao longo do tempo, seu raciocínio moral tende a evoluir (Heyman et al., 2013; Leimgruber et al., 2012).

No que diz respeito às variáveis ambientais e genéticas, ambas assumem uma parcela importante na expressão do comportamento altruísta infantil, sendo estas associadas à maior ou menor frequência de tais comportamentos. De acordo com a vertente interacionista, de Huffman et al. (2003), o comportamento humano reflete a combinação de características biológicas e culturais. Os estudos de Avinun et al. (2011) apontaram para tal interação na manifestação do comportamento altruísta. Em termos culturais (Avinun et al., 2011; Bakermans-Kranenburg \& Ijzendoorn, 2011), considera-se que, se a cultura em que a criança está inserida enfatiza o altruísmo, é mais provável que as crianças se manifestem altruístas em interações futuras.

\section{CONSIDERAÇÕES FINAIS}

A análise dos resultados apontados nos artigos que compuseram a presente revisão sistemática permite afirmar que crianças ainda muito jovens demonstram altruísmo em suas ações e à medida que suas relações crescem em número e complexidade. Múltiplas variáveis vêm sendo estudadas em associação ao altruísmo, tais como custo comportamental, sensibilidade à justiça, empatia, gerenciamento de impressão, renda familiar, distância e dominância social, respostas frente a eventos adversos, representações de apego, composição genética, entre outras.
A oportunidade parece ser o elo comum entre todas elas. É em face às oportunidades de interação oferecidas que as crianças podem vir a expressar maior ou menor frequência de altruísmo.

Além disso, embora alguns estudos de natureza biológica se dediquem a procurar explicações orgânicas para a expressão altruísta nos seres humanos, a interação entre natureza e cultura, ou seja, entre os aspectos biológicos e culturais, tem papel muito importante na emissão de comportamentos altruístas. 
Logo, se há evidências da forte influência do meio nas respostas altruístas e de que as crianças desde muito cedo são capazes de assim se comportarem, torna-se produtivo pensar em práticas educativas que visem ao estabelecimento e à manutenção do altruísmo enquanto comportamento moral. Dito de outra maneira, o processo educativo pode ser organizado visando planejar contingências de reforço para atingir este fim.

O comportamento altruísta traz vantagens tanto em nível individual quanto em nível grupal, uma vez que possibilita que as crianças tornem-se sensíveis às necessidades umas das outras, comportando-se de forma solidária e respeitosa, tornando as relações ricas em qualidade e afeto, favorecendo a sobrevivência não apenas da espécie, mas também da cultura, sobretudo, de uma cultura valorosamente fraternal. O ensino de comportamentos morais torna o indivíduo apto para viver de forma harmoniosa em sociedade e, mais que isso, é fruto de uma história de aprendizagem a qual pode ser cuidadosamente planejada. Talvez $\mathrm{o}$ aspecto mais precioso e desafiador de tudo que foi discutido até aqui resida no fato de que, se há possibilidade de aprendizagem, indivíduos, quer sejam profissionais da educação ou familiares, são convidados a fazer algo sobre isso, ou seja, são instigados a assumir um posicionamento ativo, adotando práticas favoráveis a este fim.

Em virtude da escassez de estudos que visam investigar o altruísmo, sobretudo, na infância, espera-se que esta pesquisa estimule outras que visem discutir e operacionalizar ações e natureza altruísta, explorar o papel das figuras de referência da criança no processo de aquisição de comportamento altruístas, bem como investigar a importância dos contextos de desenvolvimento da criança em estabelecer contingências promotoras de oportunidades de aquisição e manutenção do altruísmo enquanto comportamento moral. Também seria fundamental promover estudos empíricos longitudinais voltados ao ensino de comportamentos altruístas a fim de investigar a diminuição de comportamentos de natureza antissocial, bem como identificar em qual intensidade tal ensino contribui para um maior ajustamento psicossocial da criança.

\section{REFERÊNCIAS}

Aguilar-Pardo, D., Martínez-Arias, R., \& Colmenares, F. (2013). The role of inhibition in young children's altruistic behaviour. Cognitive Processing, 14 (3), 301-307. https://doi.org/10.1007/ s10339-013-0552-6

Allen-Arave, W., Gurven, M., \& Hill, K. (2008). Reciprocal altruism, rather than kin selection, maintains nepotistic food transfers on an ache reservation. Evolution and Human Behavior, 29 (5), 305-318. https://doi.org/10.1016/j.evolhumbehav.2008.03.002

Avinun, R., Israel, S., Shalev, I., Gritsenko, I., Bornstein, G., Ebstein, P. R., \& Knafo, A. (2011). AVPR1A Variant Associated with Preschoolers' Lower Altruistic Behavior. Plos ONE, 6 (9), 1-5. https://doi.org/10.1371/journal.pone.0025274

Azevedo, R. S. (2010). Sobrecarga do cuidador informal da pessoa idosa frágil: uma revisão sistemática da literatura (Dissertação de Mestrado em Enfermagem). Universidade Federal de Minas Gerais, Belo Horizonte, MG, Brasil. http://hdl.handle.net/1843/ GCPA-8D9LAL

Baum, W. M. (2006). Compreender o Behaviorismo: Comportamento, cultura e evolução. (2a. ed.) Porto Alegre: Artmed.

Bakermans-Kranenburg, M. J., \& Ijzendoorn, M. H. V. (2011). Differential susceptibility to rearing environment depending on dopamine-related genes: new evidence and a meta-analysis. Development and Psychopathology, 23 (1), 39-52. https://doi. org/10.1017/S0954579410000635

Barragan, R. C., \& Dweck, C. S. (2014). Rethinking natural altruism: simple reciprocal interactions trigger children's benevolence. Proceedings of the National Academy of Sciences of the United States of America, 111(48), 17071-17074. https:// doi.org/10.1073/pnas.1419408111

Bartholomeu, D., Silva, M. C. da R., \& Montiel, J. M. (2011). Teste de habilidades sociais para crianças: evidências psicométricas de uma versão inicial. Psico-USF, 16, 33-43. https://doi. org/10.1590/S1413-82712011000100005.

Batson, C. D., \& Powell, A. A. (2003). Altruism and prosocial behavior. In T. Millon \& M. J Lerner (Eds.), Handbook of psychology: personality and social psychology (pp 463-484), 5a ed., New York: John Wiley\& Sons.

BIREME / OPAS / OMS (2019). Biblioteca Virtual em Saúde. Thesaurus - Terminologia em Psicologia e Ciências da Saúde.
São Paulo: Disponível em: < http://brasil.bvs.br/ >.Acesso em 20 de agosto de 2019.

Bijou, S. W. (1995). Behavior Analysis of Child Development. Reno: Context Press.

Chen, Y., Zhu, L., \& Chen, Z. (2013). Family income affects children's altruistic behavior in the dictator game. Plos ONE, 8 (11), 1-8. https://doi.org/10.1371/journal.pone.0080419

Chernyak, N., \& Kushnir, T. (2013). Giving preschoolers choice increases sharing behavior. Association for Psychological Science, 24 (10), 1-9. https://doi.org/10.1177/0956797613482335

Chou, K. L. (1996). The Rushton, Chrisjohn and Fekken Selfreport altruism scale: a Chinese translation. Personality and Individual Differences, 21 (2), 297-298. https://doi. org/10.1016/0191-8869(96)00040-2

De Backer, C. J., Fisher, M. L., Poels, K., \& Ponnet, K. (2015). "Our" food versus "my" food. Investigating the relation between childhood shared food practices and adult prosocial behavior in Belgium. Appetite, 84, 54-60. http://dx.doi. org/10.1016/j.appet.2014.09.022

Dias, M. F. (2016). Estilos de liderança de Professores e comportamento acadêmico dos alunos (Dissertação de Mestrado em Educação). Universidade Federal do Paraná, Curitiba, PR, Brasil. https://acervodigital.ufpr.br/handle/1884/43270

Falcone, E. M. O., Ferreira, M. C., Luz, R. C. M., Fernandes, C. S., Faria, C. A., D’Augustin, J. F., Sardinha, A., \& Pinho, V. D. (2008). Inventário de empatia (I.E): desenvolvimento e validação de uma medida brasileira. Avaliação Psicológica, 7 (3), 321-334. Retrieved from https://bit.ly/2KDPXOZ

Ferreira, A. B. H. (1999). Aurélio século XXI: o dicionário da Língua Portuguesa, 3a. ed., Rio de Janeiro: Nova Fronteira.

Ferreira, M. F. F., \& Carrara. K. (2009). Implicações do conceito de cidadania de professores sobre comportamentos pró-éticos de estudantes. Psicol. Argum, 27 (58), 219-229. Retrieved from https://bit.ly/2Y73E0J

Flouri, E., \& Sarmadi, Z. (2015). Prosocial behavior and childhood trajectories of internalizing and externalizing problems: the role of neighborhood and school contexts. Developmental Psychology, 52 (2), 253-258. https://doi.org/10.1037/ dev0000076 
Fu, G., Heyman, G. D., Chen, G., Liu, P., \& Lee, K. (2014). Children trust people who lie to benefit others. Journal of Experimental Child Psychology, 129, 127-139. https://doi.org/10.1016/j. jecp.2014.09.006

Guimarães, R.K., \& Laurenti, C. (2019). O estudo do altruísmo na Análise do Com-portamento: um panorama a partir de uma revisão de literatura. Revista Brasileira de Terapia Comportamental e Cognitiva, 21 (3) 1-7. https://doi. org/10.31505/rbtcc.v21i3.1305

Heyman, G., Barner, D., Heumann, J., \& Schenck, L. (2013). Children's sensitivity to ulterior motives when evaluating prosocial behavior. Cognitive Science, 38 (4), 683-700. https:// doi.org/10.1111/cogs.12089

Huffman, K., Vernoy, M., \& Vernoy, J. (2003). Psicologia. São Paulo: Editora Atlas.

INEP - Instituto Nacional de Estudos e Pesquisas Educacionais Anísio Teixeira (2011). Thesaurus Brasileiro da Educação. Acesso em 20 de agosto de 2019.

Koller, S. H., Couto, M. C. P de P., \& Hohendorff, J. V. (2004). Manual de produção científica. São Paulo: Penso Editora.

Krueger, R. F., Hicks, B. M., \& Mcgue, M. (2001). Altruism and antissocial behavior: Independent tendencies, unique personality correlates, distinct etiologies. Psychology Science, 12 (5), 397-402. https://doi.org/10.1111/1467-9280.00373

Leimgruber, K. L., Shaw, L., Santos, L. R., \& Olson, K. R. (2012). Young children are more generous when others are aware of their actions. Plos ONE, 7(10), 1-8. https://doi.org/10.1371/ journal.pone. 0048292

Lencastre. M. P. A. (2010). Bondade, Altruísmo e Cooperação. Considerações evolutivas para a educação e a ética ambiental. Revista Lusófona de Educação, 15, 113-124. Retrieved from http://goo.gl/GcOsb3

Li, Y., Li, H., Decety, J., \& Lee, K. (2013). Experiencing a Natural Disaster Alters Children's Altruistic Giving. Association for Psychological Science, 24 (9), p. 1686-1695. https://doi. org/10.1177/0956797613479975

Moñivas, A. (1996). La Conduta Prosocial. Cuadernos de Trabajo Social, Madrid, 9, 125-142. Retrieved from http://goo.gl/yzIdzP

Moreira, M. B., \& Medeiros. C. A. (2008). Princípios Básicos de Análise do Comportamento. Porto Alegre: Artmed.

Nahra. C. (2016). Moral enhancement e neuroética: uma revisão da literatura neurocientífica sobre os mecanismos neurais do altruísmo. Unisinos Journal of Philosophy, 17 (3), 384-389. https://10.4013/fsu.2016.173.17

Ostrov, J. M., \& Guzzo, J. L. (2015). Prospective associations between prosocial behavior and social dominance in early child hood: are sharers the best leaders? The Journal of Genetic Psychology, 176 (2), 130-138. https://doi.org/10.1080/00221325.2015.1018860

Rex, R. V. V. (2016). O Debate sobre o nativismo moral: desenvolvimento e evolução da moralidade (Dissertação de
Mestrado em Filosofia). Universidade de Brasília, Brasília, DF, Brasil. https://repositorio.unb.br/handle/10482/22935

Rodrigues, A., Assmar, E., \& Jablonski, B. (1999). Psicologia Social. (27a. ed.) Petrópolis: Vozes.

Sabbag, G. M. (2017). Práticas educativas maternas e comportamento pró-social infantil (Tese de Doutorado em Psicologia). Universidade Federal de Santa Catarina, Florianopólis, SC, Brasil. https://repositorio.ufsc. $\mathrm{br} / \mathrm{xmlui} / \mathrm{bitstream} / \mathrm{handle} / 123456789 / 180430 / 348089$. pdf?sequence $=1 \&$ is Allowed $=y$

Sampaio, L. R., \& Carmino, C. P. S. (2017). Ajudar o próximo ou ser recompensado? Comportamento distributivo infantil em situações envolvendo mérito e altruísmo. Psico, 48(3), 186-194. https://doi.org/10.15448//1980-8623.2017.3.25746

Santos. R. B. (2017). Ética: normas e princípios para uma sociedade mais empática. Id on Line Multidisciplinary and Psycology Journal, 11 (34), 91-103. https://doi.org/10.14295/idonline. v11i34.678

Sebastián-Enesco, C., \& Warneken, F. (2015). The shadow of the future: 5-Year-olds, but not

3-year-olds, adjust their sharing in anticipation of reciprocation. Journal of Experimental Child Psychology, 129, 40-54. https:// doi.org/10.1016/j.jecp.2014.08.007

Schlinger. J. H. D. (1995). A Behavior Analytic View of Child Development. Nova York: Plenum Press.

Schmidt, M. F. H., \& Sommerville, J. A. (2011). Fairness expectations and altruistic sharing in 15-month-old human infants. Plos ONE, 6 (10), 1-9. https://doi.org/10.1371/journal. pone. 0023223

Silva, E. F., \& Moreira, A. S. A. (2019). Aproximações com os conceitos de valores, moral e ética a partir da ontologia do ser social. Anais do $16^{\circ}$ Congresso Brasileiro de Assistentes Sociais. Brasília, DF. https://broseguini.bonino.com.br/ojs/ index.php/CBAS/article/view/1188

Skinner, B. F. (1979/2000). Ciência e Comportamento Humano, 10. Ed., São Paulo: Martins Fontes (trabalho original publicado em 1953).

Sober, E., \& Wilson, D. S. (1999). Unto others: The evolution and Psychology of Unselfish Behavior. Cambridge: Harvard University Press.

Vásquez, A. S. (1995). Ética. $15^{\circ}$ edição. Rio de Janeiro: Civilização Brasileira.

Vilalva, S., \& Lohr, S. S. (2019). Comportamento altruísta em crianças de dois a cinco anos de idade. Zero-a-Seis, 21 (39), 149-165. https://doi.org/10.5007/1980-4512.2019v21n39p149

Warneken, F., \& Tomasello, M. (2011). The roots of human altruism. British Journal of Psychology, 100 (3), 455-471. https://doi. org/10.1348/000712608X379061

Weiszflog, W. (2004). Michaelis Moderno Dicionário da Língua Portuguesa. São Paulo: Melhoramentos. 